\title{
Expression of vacuole membrane protein 1 (VMP1) in spontaneous chronic pancreatitis in the WBN/Kob rat
}

\author{
Pei-Hong Jiang, Yoshiharu Motoo, Gensaku Okada, and Norio Sawabu
}

VMP1 is a transmembrane protein located in the Golgi and endoplasmic reticulum. It is strongly and rapidly induced in pancreas during acute pancreatitis whereas it is hardly detectable in normal rats pancreas. Its overexpression promotes formation of intracellular vacuoles and cell death through apoptosis. Intracellular vacuolation is a cellular response to injury in the pancreas during pancreatitis to maintain function under stress. The vacuoles have been shown to contain both digestive and lysosomal enzymes suggesting an abnormal intracellular processing of newly synthesized enzymes in acute pancreatitis. Physiologically, digestive and lysosomal enzymes are synthesized in ribosomes attached to the rough endoplasmic reticulum. Vacuoles represent early morphological changes during apoptosis. The aim of this work was to examine the kinetics of VMP1 gene expression in spontaneous chronic pancreatitis in WBN/Kob rat.

Four-week-old male WBN/Kob rats were fed a special breeding diet MB-3 to induce chronic pancreatitis. Sections were stained with hematoxylin and eosin for histological evaluation and vacuoles examination. VMP1 mRNA expression was determined by RT-PCR with a semi-quantitative analysis and in situ hybridization. TUNEL was used to detect cell apoptosis. Cytoplasmic vacuoles with different sizes were observed in the pancreas of the WBN/Kob rat from 4 to 24 weeks. The vacuoles increased in size and number at the onset of CP at 12 weeks. VMP1 mRNA expression began at 8 weeks, reached a peak at 12 weeks, decreased at 16 and 20 weeks, then disappeared at 24 weeks. It was showed VMP1 mRNA was located in acinar cells and absent in the islets of Langerhans, duct cells, inflammatory infiltrates, and other stromal cells by in situ hybridization analysis. The vacuoles formation was paralleled to VMP1 mRNA expression. The kinetics of VMP1 mRNA expression also corresponded to the apoptosis of acinar cells.

Vacuolation is an early phenomenon proceeding cell death, and is related to apoptosis. We have shown, for the first time, that cytoplasmic vacuoles in acinar cells are increased during the course of $\mathrm{CP}$ in the $\mathrm{WBN} / \mathrm{Kob}$ rat and that the number of vacuoles was most increased at the onset of the CP. Acinar cell vacuolation is possibly a general feature of the development of acute pancreatitis and therefore, may also have important pathophysiological implications for CP. VMP1 gene activation in chronic pancreatitis is part of the acinar cell response to aggression. In conclusion, we have revealed cytoplasmic vacuolization in CP of the WBN/Kob rat, and demonstrated that VMP1 mRNA expression is strongly induced at the onset of CP. VMP1 mRNA expression, relating to acinar cell apoptosis, may reflect important pathophysislogical
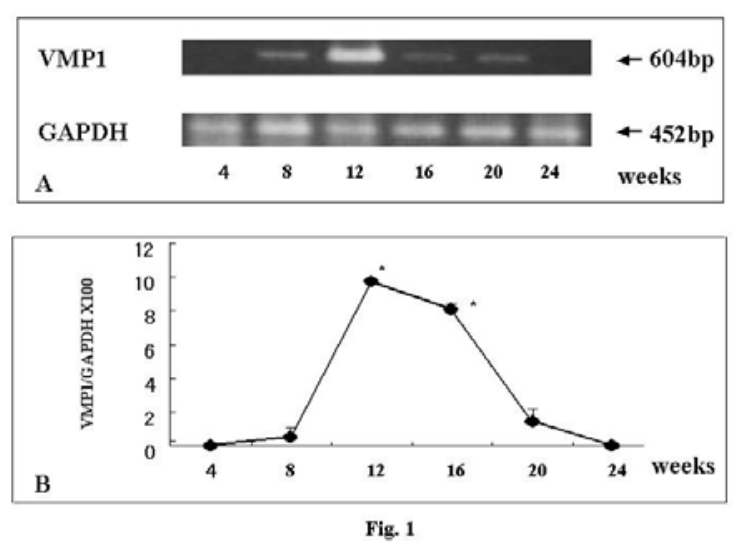
changes in $\mathrm{CP}$.

Figure: A: VMP1 mRNA of $604 \mathrm{bp}$ was expressed from 8 to 20 weeks. B: VMP1 mRNA expression began at 8 weeks reached its peak at 12 weeks decreased at 16 and 20 weeks. 